Case Reports

\title{
Disseminated Fusarium Infections in Patients Receiving Posaconazole Prophylaxis: A Report of 2 Patients and Brief Review of the Literature
}

\author{
${ }^{1}$ John Byrne Fournier, ${ }^{2}$ Vinod Thomas, ${ }^{3}$ Catherine Breen, \\ ${ }^{4}$ Jonathan Cannella, ${ }^{1}$ Douglas Heiner, ${ }^{5}$ Todd Roberts and ${ }^{6}$ Gail Skowron \\ ${ }^{I}$ Department of Dermatology, \\ ${ }^{2,6}$ Department of Infectious Diseases, \\ ${ }^{3,4}$ Department of Pathology, \\ ${ }^{5}$ Department of Oncology and Hematology, \\ M.D.Roger Williams Medical Center/Boston University School of Medicine, United States
}

\author{
Article history \\ Received: 05-04-2015 \\ Revised: 15-09-2015 \\ Accepted: 21-10-2015 \\ Corresponding Author: \\ John Byrne Fournier \\ M.D.Roger Williams Medical \\ Center/Boston University \\ School of Medicine, United \\ States \\ Tel: 4014562521 \\ E-mail: Johnbfournier@gmail.com
}

\begin{abstract}
Fusarium is a filamentous fungi that has emerged as a frequent cause of invasive infections in immunocompromised patients. Dermatologists often play a critical role in diagnosing fusariosis, as greater than $70 \%$ of patients with disseminated infections have cutaneous involvement, and microbiologic evaluation of skin specimens provides the diagnosis in the majority of cases. Second-generation triazoles are typically the most effective medications in treating invasive Fusarium infections, and we discuss the benefits of using these medications, as well as detail the side effects of these therapeutic agents.
\end{abstract}

Keywords: Fusarium, Invasive Fungal Infection, Posaconazole, Voriconazole

\section{Introduction}

Fusarium is a saprophytic filamentous fungi belonging to the Nectriaceae family and has a worldwide distribution. Infections occurring in immunocompetent hosts are usually localized and typically follow trauma or occur at sites of pre-existing onychomycosis (Nucci and Anaissie, 2002). Although infections in immunocompetent patients are generally superficial and non-life-threatening, Fusarium has emerged as a frequent cause of invasive fungal infections in immunocompromised patients (Nucci and Anaissie, 2002; 2007). Unfortunately, disseminated Fusarium infections have historically been associated with high rates of mortality, although second-generation triazoles have demonstrated promising therapeutic results in recent years (Nucci and Anaisse, 2007; Labois et al., 2011; Perfect et al., 2003). We present two cases of disseminated Fusarium infections and both infections occurred in patients who were receiving fungal prophylaxis with posaconazole.

\section{Case 1}

A 64-year-old male, with a five-month history of Acute Myeloid Leukemia (AML), presented to the emergency department with hemoptysis, chest pain, shortness of breath, and a 1-week history of an indurated nodule on the left anterior thigh, an erythematous patch over the left lateral malleolus, an erythematous papule on the left cheek, and several necrotic eschars on the scrotum. On physical examination, a $6 \times 4 \mathrm{~cm}$ erythematous nodule with central necrosis was present on the left anterior thigh (Fig. 1), as well as twelve necrotic ulcers on the scrotum (Fig. 2), a $7 \mathrm{~mm} \times 5 \mathrm{~mm}$ erythematous papule with central necrosis on the left cheek, and a $8 \times 10 \mathrm{~cm}$ indurated erythematous patch over the left lateral malleolus. All lesions were exquisitely tender to palpation. Two weeks prior to presentation, he had completed a course of FLAG chemotherapy (fludarabine, cytarabine, and granulocytecolony stimulating factor (G-CSF)) for acute myeloid leukemia. Past medical history also included a suspected left upper lobe aspergilloma (fungal smear and fungal culture of bronchoscopy specimens were negative), which was diagnosed three months prior to presentation, and subsequent imaging showed resolution of the lung mass following treatment with posaconazole. In addition to recently receiving chemotherapy, he was taking several prophylactic medications, including posaconazole $(200 \mathrm{mg}$ q8 h), trimethoprim-sulfamethoxazole, and acyclovir.

In the emergency department a Non-ST Elevation Myocardial Infarction (NSTEMI) was diagnosed and he was transferred to the intensive care unit. Laboratory examination revealed a White Blood Cell (WBC) count of $0.2 \times 10^{3} / \mu \mathrm{L}$, with $4 \%$ segmented neutrophils, $0 \%$ bands, 
and an Absolute Neutrophil Count (ANC) of 8 cells $/ \mu \mathrm{L}$. Computed Tomography (CT) imaging of the chest revealed a cavitary lesion of the left upper lobe, as well as bilateral lung nodules. Although he had received prophylaxis with posaconazole, cutaneous findings and CT imaging were concerning for a disseminated fungal infection. Given the central necrosis of the cutaneous lesions, particular concern was for Aspergillosis, Mucormycosis, or Fusarium, and this prompted empiric treatment with voriconazole and amphotericin B. Microscopic examination of fluid and tissue from the necrotic pustule on the left cheek were negative for fungal elements, however, both cultures grew a filamentous fungus that was identified as Fusarium solani, which was sensitive to voriconazole and amphotericin B. Skin biopsy of the left thigh revealed dermal fibrosis with hemorrhage, edema, and a lymphohistiotic infiltrate with underlying fat necrosis. No organisms were identified on Periodic Acid-Schiff (PAS) or GomoriGrocott Methenamine Silver (GMS) stains. Aspergillus antigen (galactomannan) testing with an enzyme immunoassay was negative, although $1,3-\beta$-D-Glucan assay was positive $(101 \mathrm{pg} / \mathrm{ml}$, positive value $>80 \mathrm{pg} / \mathrm{mL})$. A bronchoscopy was performed, and microbiologic tests were negative on bronchial washings and included fungal smear, fungal culture, gram stain, bacterial culture, acid-fast stain, and Acid-Fast Bacilli (AFB) culture.

A diagnosis of a disseminated Fusarium infection was made. He continued intravenous voriconazole for ten days, before being transitioned to oral voriconazole. Six weeks after the initial presentation he was doing well, and lesions on the left cheek, left lateral malleolus, and scrotum had completely resolved, and the lesion on the left anterior thigh had improved considerably. Following several weeks of G-CSF administration, the neutropenia had also resolved (ANC 3200 cells $/ \mu \mathrm{L}$ ). Unfortunately, three months after the initial presentation, he died following another cardiac event.

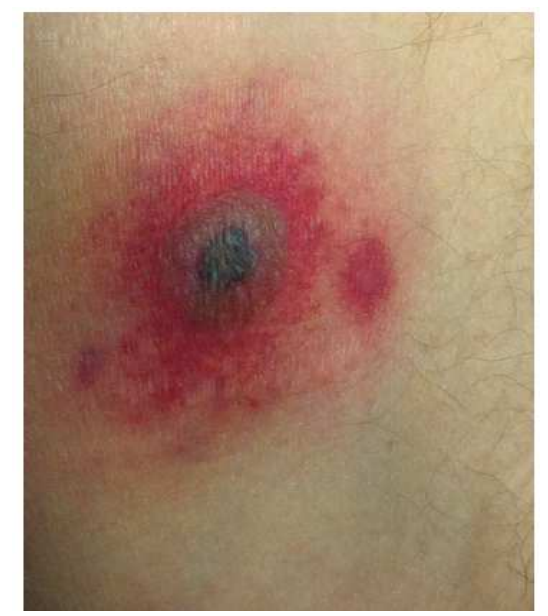

Fig. 1. A $6 \times 4 \mathrm{~cm}$ erythematous nodule with central necrosis was present on the left anterior thigh

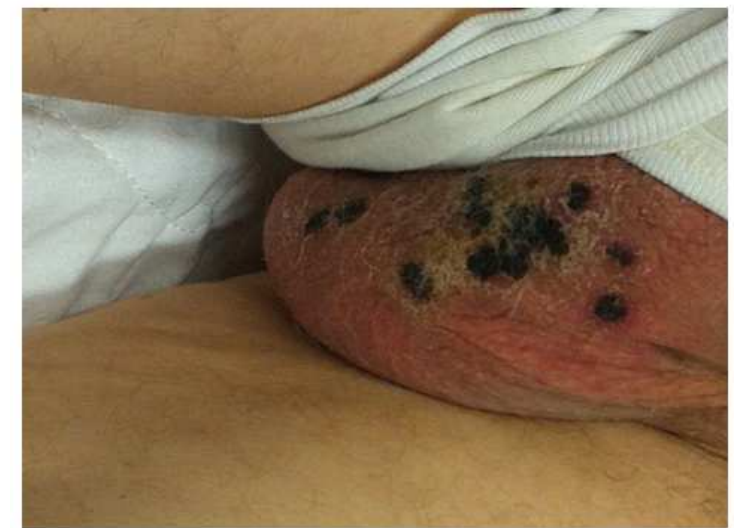

Fig. 2. 12 Necrotic ulcers on the scrotum

\section{Case 2}

A 60-year-old male, with a five-year history of acute myeloid leukemia, developed an erythematous nodule with central necrosis on his right index finger, during a hospital admission. The patient had received an allogenic stem cell transplant 5 years prior to his admission, although he had recently relapsed, and he was receiving etoposide and decetabine for treatment of AML.

Two weeks priors prior to the development of the erythematous nodule, the patient had received a small laceration on his right index finger, following accidental trauma with the showerhead in his hospital bathroom. The nodule developed a central eschar and fungal culture grew Fusarium dimerum. At the time of the Fusarium diagnosis, the patient had a WBC of $0.9 \times 10^{3} / \mu \mathrm{L}$, with an ANC 0.0 cells $/ \mu \mathrm{L}$.

Although he had been receiving fungal prophylaxis with posaconazole (dose $200 \mathrm{mg} \mathrm{q} 8 \mathrm{~h}$ ), following the positive Fusarium culture, the posaconazole was discontinued and voriconazole was started. A morbilliform drug eruption developed, however, and the patient was transitioned back to posaconazole (re-started at a therapeutic dosage; $400 \mathrm{mg}$ q8 h) and amphotericin B was also started at this time. Two months later (while still receiving treatment with posaconazole and amphotericin B), an erythematous nodule with central necrosis developed on the shaft of his penis and culture grew Fusarium. The patient then developed pain and swelling of the left eye and was diagnosed with fungal endophthalmitis, and a vitrectomy was performed.

The patient continued on posaconazole and amphotericin B for an additional four months following vitrectomy (amphotericin dosing at $5 \mathrm{mg} / \mathrm{kg}$, three times weekly), during which time the skin and eye lesions resolved, and his ANC recovered following cessation of chemotherapy (ANC 3700 cells/ $\mu \mathrm{L}$ ). Eight months after the initial lesion appeared on the hand, there is no evidence of Fusarium infection. 
Comment. Fusarium infections are a significant cause of morbidity and mortality in immunocompromised patients (Nucci and Anaissie, 2002; 2007). At highest risk for developing infections are those with hematologic malignancies, bone marrow transplant recipients, and patients receiving chemotherapy (Nucci and Anaissie, 2002 ; 2007). Moreover, any medical condition that causes neutropenia likely increases the risk for developing a disseminated Fusarium infection, as neutrophils bind to hyphae and facilitate killing through the secretion of reactive oxygen species (Sixto et al., 2012; Sun et al., 2010).

Portals of entry for Fusarium include the respiratory tree, gastrointestinal tract, through venous catheters, and at sites of compromised skin integrity (Nucci and Anaisse, 2007; Gupta et al., 2000; Boutati and Anaisse, 1997). Although skin can serve as a portal of entry, as Fusarium is angioinvasive and can spread hematogenously, most cases of disseminated infection originate in the respiratory system (Carneiro et al., 2011). Despite the fact that skin is not typically the site of primary infection, cutaneous involvement is common in disseminated fusariosis, and skin lesions are present in greater than $70 \%$ of patients (Nucci and Anaissie, 2002). Several morphological variants of cutaneous Fusarium have been described, although painful erythematous papules or nodules are most common, and many lesions have an eschar or central necrosis (Nucci and Anaisse, 2007; Bodey et al., 2002). Even when characteristic skin lesions are present, however, Fusarium is often clinically indistinguishable from Aspergillosis. Moreover, both Fusarium and Aspergillosis have septate hyphae with acute-angle-branching, and are also typically indistinguishable on histopathology (Lionakis and Kontoyiannis, 2004; Liu et al., 1998). Although a PCR technique has been developed to detect Fusarium, culture remains the gold standard for diagnosis (Hue et al., 1999; Fan et al., 2010).

Treatment of Fusarium and Aspergillosis infections previously differed, thus necessitating the importance of differentiating between the filamentous fungi in a timely manner, although both are now most-effectively treated with second-generation triazoles (Herbrecht et al., 2002). Voriconazole is a second-generation triazole that was approved by the US Food and Drug Administration (FDA) in 2002 for the treatment of invasive Fusarium and Aspergillosis infections (Pemán et al., 2006). Voriconazole is generally well-tolerated and the most frequently encountered side-effects are transient visual disturbances, although cutaneous reactions are also common, and include chelitis and increased photosensitivity (Johnson and Kauffman, 2003; Racette et al., 2005; McCarthy et al., 2007; Cowen et al., 2010). Although increased photosensitivity is a well-described side effect, and long-term voriconazole treatment is a risk factor for developing squamous cell carcinoma of the skin, Miller et al. (2010) also recently reported five cases of in situ melanoma occurring in patients treated with voriconazole (Vadnerkar et al., 2010; Miller et al., 2010). Therefore, it is imperative that all patients who are receiving voriconazole therapy receive regular full-body skin examinations. Overall, second-generation triazoles have a favorable side-effect profile and are more effective than previous treatments, as the cure rate with voriconazole approaches fifty-percent (Perfect et al., 2003).

While voriconazole has become the first-line treatment for invasive Fusarium infections, another secondgeneration triazole, posaconazole, has demonstrated superiority over fluconazole for fungal prophylaxis in patients with hematologic malignancies. In addition to a reduced incidence of invasive fungal infections, posaconazole has been associated with an overall survival benefit when compared with fluconazole (Cornely et al., 2007). Although posaconazole is more effective at preventing invasive fungal infections than fluconazole, several cases of disseminated fusariosis have been reported in patients receiving prophylaxis with posaconazole (Bose et al., 2011). Studies have shown that posaconazole serum levels are often subtherapeutic despite appropriate dosing, and taking the medication with a high-fat meal may maximize absorption (Thompson et al., 2009).

Although second-generation triazoles are typically the most effective medications in treating Fusarium infections, recovery from neutropenia remains the most significant prognostic factor, and persistently neutropenic patients have mortality rates of nearly $100 \%$ (Nucci and Anaisse, 2007). While the benefits of G-CSF remain unclear in Fusarium infections, it may serve as an important adjunctive therapy. While the use of G-CSF leads to accelerated recovery from neutropenia and thus may lead to improved outcomes, Fusarium spp. will likely continue to be a common cause of opportunistic infections, due to the frequency of stem cell transplants being performed and the need for immunosuppressive medications.

\section{Author's Contributions}

All authors contributed to the writing of this manuscript.

\section{Ethics}

This article is original and contains unpublished material. The corresponding author confirms that all of the other authors have read and approved the manuscript and no ethical issues involved.

\section{References}

Bodey, G., M. Boktour, S. Mays, M. Duvic and D. Kontoyiannis et al., 2002. Skin lesions associated with Fusarium infection. J. Am. Acad. Dermatol., 47: 659-66. DOI: 10.1067/mjd.2002.123489 
Bose, P., H.D. Parekh, J.L. Holter and R.A. Greenfield, 2011. Disseminated fusariosis occurring in two patients despite posaconazole prophylaxis. J. Clin. Microbiol., 49: 1674-1675. DOI: 10.1128/JCM.02325-10

Boutati, E.I. and E.J. Anaisse, 1997. Fusarium, a significant emerging pathogen in patients with hematologic malignancy: Ten years' experience at a cancer center and implications for management. Blood, 90: 999-1008. PMID: 9242529

Carneiro, H.A., J.J. Coleman, A. Restrepo and E. Mylonakis, 2011. Fusarium infection in lung transplant patients: Report of 6 cases and review of the literature. Medicine (Baltimore), 90: 69-80.

Cornely, O.A., J. Maertens, D.J. Winston, J. Perfect and A.J. Ullmann et al., 2007. Posaconazole Vs. fluconazole or itraconazole prophylaxis in patients with neutropenia. N Engl. J. Med., 25: 348-59.

DOI: 10.1056/NEJMoa061094

Cowen, E.W., J.C. Nguyen, D.D. Miller, D. McShane and S.T. Arron et al., 2010. Chronic phototoxicity and aggressive squamous cell carcinoma of the skin in children and adults during treatment with voriconazole. J. Am. Acad. Dermatol., 62: 31-7. DOI: $10.1016 /$ j.jaad.2009.09.033

Fan, Y., L. Willems, C. Leboeuf, W. Li and C. Lacroix et al., 2010. Skin microvascular thrombosis in fusarium infection in two early biopsied cases. Case Rep Dermatol., 2: 76-81. DOI: 10.1159/000313934

Gupta, A.K., R. Baran and R.C. Summerbell, 2000. Fusarium infections of the skin. Curr Opin Infect. Dis., 13: 121-128.

Herbrecht, R., D.W. Denning, T.F. Patterson, J.E. Bennett and R.E. Greene et al., 2002. Voriconazole versus amphotericin B for primary therapy of invasive aspergillosis. N Engl. J. Med., 347: 408-15. DOI: 10.1056/NEJMoa020191

Hue, F.X., M. Huerre, M.A. Rouffault and C. de Bievre, 1999. Specific detection of fusarium species in blood and tissues by a PCR technique. J. Clin. Microbiol., 37: 2434-8.

Johnson, L.B. and C.A. Kauffman, 2003. Voriconazole: A new triazole antifungal agent. Clin. Infect. Dis., 36: 630-637. DOI: 10.1086/367933

Labois, A., C. Gray and S. Lepetre, 2011. Successful treatment of disseminated fusariosis with voriconazole in an acute lymphoblastic leukaemia patient. Mycoses, 4: 8-11. DOI: 10.1111/j.1439-0507.2011.02136.x

Lionakis, M.S. and D.P. Kontoyiannis, 2004. Fusarium infections in critically ill patients. Semin Respir Crit Care Med., 25: 159-69. DOI: 10.1055/s-2004-824900

Liu, K., D.N. Howell, J.R. Perfect and W.A. Schell, 1998. Morphologic criteria for the preliminary identification of Fusarium, Paecilomyces and Acremonium species by histopathology. Am. J. Clin Pathol., 109: 45-54.
McCarthy, K.L., E.G. Playford, D.F. Looke and M. Whitby, 2007. Severe photosensitivity causing multifocal squamous cell carcinomas secondary to prolonged voriconazole therapy. Clin. Infect. Dis., 44: e55-56. DOI: $10.1086 / 511685$

Miller, D.D., E.W. Cowen, J.C. Nguyen, T.H. McCAlmont and L.P. Fox, 2010. Melanoma associated with longterm voriconazole therapy: A new manifestation of chronic photosensitivity. Arch Dermatol., 146: 300-4. DOI: 10.1001/archdermatol.2009.362

Nucci, M. and E. Anaisse, 2007. Fusarium infections in immunocompromised patients. Clin. Microbiol. Rev., 20: 695-704. DOI: 10.1128/CMR.00014-07

Nucci, M. and E. Anaissie, 2002. Cutaneous infection by Fusarium species in healthy and immunocompromised hosts: Implications for diagnosis and management. Clin. Infect. Dis., 15: 909-20. DOI: 10.1086/342328

Pemán, J., M. Salavert, E. Cantón, I. Jarque and E. Romá et al., 2006. Voriconazole in the management of nosocomial invasive fungal infections. Ther Clin. Risk Manag., 2: 129-158.

Perfect, J.R., K.A. Marr, T.J. Walsh, R.N. Greenberg and B. DuPont et al., 2003. Voriconazole treatment for lesscommon, emerging, or refractory fungal infections. Clin. Infect. Dis., 36: 1122-31. DOI: 10.1086/374557

Racette, A.J., H.H. Roenigk Jr., R. Hansen, D. Mendelson and A. Park, 2005. Photoaging and phototoxicity from long-term voriconazole treatment in a 15-year-old girl. J. Am. Acad. Dermatol., 52: S81-5. PMID: 15858516

Sixto, L.M., C. Vareechon, S. Cowden, B. Cobb and J. Latge et al., 2012. Fungal Antioxidant pathways promote survival against neutrophils during infection. J. Clin. Invest., 122: 2482-2498.

Sun, Y., J. Chandra, P. Mukherjee, L. Szczotka-Flynn and M.A. Ghannoum et al., 2010. A murine model of contact-lens-associated Fusarium keratitis. Invest Ophthalmo Vis Sci., 51: 1511-1516. DOI: $10.1167 /$ iovs.09-4237

Thompson, G.R., M.G. Rinaldi, G. Pennick, S.A. Dorsey and T.F. Patterson et al., 2009. Posaconazole therapeutic drug monitoring: A reference laboratory experience. Antimicrobial Agents Chemotherapy, 53: 2223-4. DOI: 10.1128/AAC.00240-09

Vadnerkar, A., M.H. Nguyen, D. Mitsani, M. Crespo and J. Pilewski et al., 2010. Voriconazole exposure and geographic location are independent risk factors for squamous cell carcinoma of the skin among lung transplant recipients. J. Heart Lung Transplant., 29: 1240-1244. DOI: 10.1016/j.healun.2010.05.022 